\title{
Low back pain
}

\section{Can we mitigate the inadvertent psycho-behavioural harms of spinal imaging?}

Luke P Wheeler, Emma L Karran, Daniel S Harvie

\section{Background \\ Low back pain is responsible for significant personal and societal burden, particularly when it becomes persistent. Despite international consensus regarding the judicious use of diagnostic spinal imaging, patients continue to be over-referred.}

\section{Objectives}

The aim of this article is to highlight the critical need for primary care clinicians to engage in thoughtful use of imaging procedures, and to consider alternative or adjunct methods for providing reassurance, in order to avoid or mitigate the potential negative impact of 'anomalous' findings.

\section{Discussion}

While imaging is frequently requested with the goal of reassuring patients, it can paradoxically have a negative impact on patient attitudes and beliefs and can influence pain behaviours. For improved patient outcomes we recommend contextualisation of radiological findings within age-related norms, use of reassuring and non-threatening language when communicating results, and educating patients on non-pathoanatomical contributors to pain.
LOW BACK PAIN (LBP) is the most common musculoskeletal complaint seen in general practice in Australia, and is believed to affect the lives of one in seven to one in four Australians at any time..$^{1,2}$ LBP is frequently benign and self-limiting; however, a significant proportion of adults with acute LBP (10-40\%) develop persistent and disabling symptoms. ${ }^{3,4}$

Spinal imaging is rarely indicated for patients presenting to primary care with a first episode of LBP, yet it is frequently requested to aid in diagnosis, rule out sinister pathology, guide treatment strategies or provide reassurance for the patient and clinician. ${ }^{4}$ However, imaging reports are not always reassuring and may even be perceived as threatening, given the high prevalence of aberrant findings. Much of the resulting concern is likely to be unwarranted, as many of the 'abnormal' features identified are likely to be found incidentally and represent 'normal', age-related changes that lack association with pain or prognosis. ${ }^{5}$

Overuse of spinal imaging, misinterpretation of the clinical relevance of incidental findings and poor communication of relevant findings are likely to have adverse consequences for individual patient outcomes and healthcare expenditure. Thus, it is important for clinicians to be aware of best practice regarding imaging for spinal pain, to understand alternative approaches to providing reassurance when imaging is unnecessary, and to have strategies for mitigating the potential negative impacts of spinal imaging in cases where imaging is nonetheless performed.

\section{Mechanisms of LBP}

For acute LBP, a specific pathoanatomical cause is identified in only $8-15 \%$ of patients; the remainder are given the arbitrary diagnosis of 'non-specific low back pain' (NSLBP). ${ }^{6}$ Patients with LBP rarely present with symptoms that directly correspond with identifiable pathology in a linear fashion, but instead portray a complex interaction of tissue-based, immune (eg inflammatory), central nervous system (eg central sensitisation) and cognitive behavioral processes. In the case of chronic LBP, central processes are thought to have a more dominant role. Indeed, some of the strongest predictors of non-recovery are non-physical factors. Often described as 'yellow flags', psychosocial factors, including depression and anxiety or sustained stress, cognitive factors such as poor recovery expectation and a catastrophic interpretation of symptoms, are all significant predictors of chronic LBP following injury. ${ }^{7}$ It is believed that these factors may contribute to pain and disability through alteration of painrelated sensory processing, dysregulation of stress-related neuroendocrine responses and behavioral responses that are less conducive to recovery - such as avoidance of movement and activity. ${ }^{8}$

\section{Imaging best practice}

International guidelines for the management of LBP are consistent in several respects (Box 1). They suggest a focus on short-term pain reduction strategies, provision of education including advice to remain active, patient 
reassurance and follow-up review to ensure return to normal function..$^{4,9}$ Of key relevance to this paper is the broad consensus that spinal imaging should not be routinely ordered.

Unfortunately, evidence-based recommendations and clinical practice do not always align; this may be detrimental to patient outcomes. For example, despite guidance regarding the use of simple analgesics, patients continue to be prescribed unduly high doses of opioids, which have recently been shown to be less effective than simple analgesia and may paradoxically increase pain sensitivity in the long term. ${ }^{9,10}$ Similarly, clinical guidelines consistently specify that imaging is only required in specific circumstances, particularly in the presence of red flags, a term widely used for signs and symptoms that may indicate serious pathology, such as spinal fracture, metastasis, infection, spondyloarthritis or cauda equina syndrome. However, further complicating the issue is the fact that referral for imaging on the suspicion of red flag pathology alone produces a high number of false positives, which prompt further assessment and clinical reasoning to appropriately determine whether imaging is required. Articles by Traeger et $\mathrm{al}^{11}$ and Atlas and Deyo ${ }^{12}$ provide more detailed recommendations. As an example, night pain, a widely accepted red flag, may be present in up to $44 \%$ of back pain cases; however, serious pathology is present in $<1 \%$ of patients. ${ }^{11}$

The decision to order spinal imaging in primary care is likely to be influenced by the patient, clinician and systemic factors. For example, general practitioners (GPs) and their patients are likely to be eager to obtain a specific diagnosis, ascertain treatment options and rule out a serious underlying cause. The latter consideration may be particularly important to clinicians who perceive themselves as vulnerable to litigation and are challenged by the need to exclude the possibility of serious pathology within short consultation times. ${ }^{13-15}$ Even in situations where the GP advises against imaging, patient expectations (along with GP perceptions of the expectations of their patients) may nonetheless lead to referral. In Australia, spinal imaging is widely available and easily organised via an abundance of private radiology companies. ${ }^{16}$ As a result, imaging is not only frequently expected by patients and preferred by clinicians but is also highly accessible, with limited accountability.

As a result of the above factors, many people are referred for spinal imaging without evidence of benefit. This is of particular interest given the increasing evidence that imaging may have adverse effects via psycho-behavioural mechanisms. ${ }^{4,9,15}$

\section{Potential negative effect of imaging}

While one of the primary aims of requesting investigations may be to reassure clinicians and patients, patients may instead be disturbed by the identification of degenerative changes. In fact, it has been shown that diagnostic testing, across multiple conditions, does not reduce patient concern for conditions or diseases with a low suspicion of serious disease, and that GPs commonly overestimate the reassuring potential of investigations in these cases. ${ }^{17,18}$ Instead, spinal imaging for NSLBP has been found to be associated with increased or prolonged disability, increased healthcare costs and a decreased sense of wellbeing. ${ }^{18}$

One possible explanation for the detrimental effect of imaging is that radiologists often report an array of abnormalities, which frequently fall within the range of age-related norms. Many common degenerative features are present in asymptomatic populations and are not robustly linked to clinical symptoms and long-term individual outcomes. ${ }^{18-20}$ However, spinal imaging is often reported in a manner that leads to misunderstanding of the relevance of the features identified, leading patients to believe that their spine is 'damaged' or vulnerable - perhaps irreversibly. These negative structure-related perceptions and beliefs may in turn lead to distress, enhanced pain via neuropsychological and neuro-immune mechanisms, and the compounding of disability through behavioural mechanisms such as fear and avoidance. ${ }^{21,22}$ Clinically, this complexity may manifest as higher levels of pain or disability than expected and a higher incidence of chronicity.

\section{Recommendations}

With best-practice guidelines readily available, clinicians can be confident that if a patient does not have any clinical signs of serious pathology (ie red flags), spinal imaging can be delayed or avoided altogether. While large allocations of time are not always available for primary care clinicians, it is important that a clinician moves focus away from a pathoanatomical focus towards a constructive understanding and positive, active-management approach. ${ }^{23}$ Findings from a preliminary investigation suggest that patients with LBP who receive high-quality information regarding their condition and no imaging tend to have higher satisfaction and more positive perceptions about their spine and recovery. ${ }^{15}$ Public education may also assist in reducing the pressure on GPs to refer for imaging - similar to state health service media campaigns designed to inform the public about appropriate use of emergency departments and alternative services. Along with curbing patient expectations for imaging, an evidencebased, self-directed, active-management approach should be promoted.

Furthermore, a systemic approach may assist in promoting best practice and reducing the volume of spinal imaging. One such example may be a system similar to the Australian Pharmaceutical Benefits Scheme for access to restricted medicines,

\section{Box 1. Guideline recommendations for the management of non-specific low back pain ${ }^{27}$}

\footnotetext{
- Exclude alternative diagnoses (ie red flags)

- Avoid routine use of imaging

- Offer high-quality education

- Encourage return to normal activity

- Encourage physical exercise

- Advise use of simple analgesia

- Use opioid analgesia cautiously
} 
whereby availability is dependent on the request meeting guideline criteria for access to services.

When imaging is performed, a number of strategies could be implemented to mitigate the potential negative impact. First, it has been suggested that radiology reporting could include data regarding normal, age-related findings in an attempt to convey the clinical relevance of the features identified and reduce the possibility of misinterpretation. ${ }^{15,24}$ This approach has been shown to be promising for lumbar magnetic resonance imaging reports and cardiac testing, with patients being reassured by 'normal results' when relevant epidemiological information is included. ${ }^{25,26}$

Second, the practitioners' communication of imaging findings is likely to have a vital impact. Clinicians play an important part in influencing patients' perceptions and subsequent behaviour; therefore, it would be beneficial to carefully consider the language used and the messages delivered when explaining imaging findings to patients. Clinicians who use negative

\section{Box 2. Important messages for patient-clinician interactions $4,9,15,18-20,24$}

- Without clinical risk factors (red flags), serious pathology is uncommon.

- Positive communication from the clinician has a significant impact on the patient.

- Most non-specific low back pain improves after 4-6 weeks, and returning to normal activity as soon as possible is highly beneficial.

- Evidence shows no benefit (and potentially negative impact) of routine imaging.

- When imaging is expected, discussions of evidence-based guidelines to aid in education may prove helpful.

- Radiological abnormalities are common and not often correlated with clinical presentation.

- Imaging findings including 'age-related changes' should be explained to the patient with epidemiological context and with non-threatening language.

- Referral to other health professionals may aid in patient education and improved recovery. terminology or fail to challenge patients' negative perceptions may be missing an opportunity to positively influence the patients' long-term outcomes. ${ }^{15}$ Clinicians should consider the use of non-threatening metaphorical labels for age-related findings (eg 'the kisses of time' or 'like wrinkles on the inside'), providing positive reinforcement (evidenced by the spinal images) of the inherent strength and stability of the spine, and providing an explanation of the complex, non-pathoanatomical contributors to pain. ${ }^{18,21}$ Again, emphasis should be deferred away from pathoanatomical causes of pain and towards active management of the condition according to best practice guidelines. Some key points to consider when communicating with clients about imaging are detailed in Box 2.

\section{Conclusion}

Counterintuitively, imaging for NSLBP has frequently been found to be associated with adverse outcomes, such as detrimental effects on disability, healthcare costs and sense of wellbeing. By contrast, patients who receive highquality information regarding their clinical presentation without imaging - and as per guideline-based recommendations - are likely to fare better. Where imaging is performed, we suggest a number of recommendations including:

- contextualisation of radiological findings within age-related norms

- use of reassuring and non-threatening language when communicating results

- educating patients on nonpathoanatomical contributors to pain.

\section{Authors}

Luke P Wheeler BAppSc (Hum Mvt), M Physio, MBBS, Northern Adelaide Local Health Network, Elizabeth Vale, SA. Lukewheels@outlook.com

Emma L Karran PhD, BAppSc (Physio) (Hons), GradDipPsych, School of Health Sciences, University of South Australia, Adelaide, SA; Royal Adelaide Hospital, Adelaide, SA

Daniel S Harvie PhD, M Musc Sports Physio, B Physio (Hons), The Hopkins Centre, Menzies Health Institute Queensland, Griffith University; School of Health Sciences, University of South Australia, Adelaide, SA Competing interests: None.

Provenance and peer review: Not commissioned, externally peer reviewed.

\section{References}

1. Bardin LD, King P, Maher CG. Diagnostic triage for low back pain: A practical approach for primary care. Med J Aust 2017;206(6):268-73.

2. Walker BF. The prevalence of low back pain in Australian adults. A systematic review of the literature from 1966-1998. Asia Pac J Public Health 1999;11(1):45-51.

3. Dillingham T. Evaluation and management of low back pain: An overview. State Art Review 1995;9:559-74.

4. Maher $\mathrm{C}$, Underwood $\mathrm{M}$, Buchinder $\mathrm{R}$. Non-specific low back pain. Lancet 2017;389(10070):736-47. doi: 10.1016/S01406736(16)30970-9.

5. Brinjikji W, Luetmer PH, Comstock B, et al. Systematic literature review of imaging features of spinal degeneration in asymptomatic populations. AJNR Am J Neuroradiol 2015;36(4):811-16. doi: 10.3174/ajnr.A4173.

6. Deyo RA, Mirza SK, Turner JA, Martin BI. Overtreating chronic back pain: Time to back off? J Am Board Fam Med 2009;22(1):62-68. doi: 10.3122/jabfm.2009.01.080102

7. Gatchel RJ, Peng YB, Peters ML, Fuchs PN, Turk DC. The biopsychosocial approach to chronic pain: Scientific advances and future directions. Psychol Bull 2007;133(4):581-624.

8. Main CJ, Foster N, Buchbinder R. How important are back pain beliefs and expectations for satisfactory recovery from back pain? Best Pract Res Clin Rheumatol 2010;24(2):205-17. doi: 10.1016/j.berh.2009.12.012.

9. Williams CM, Maher CG, Hancock MJ, et al. Low back pain and best practice care: A survey of general practice physicians. Arch Intern Med 2010;170(3):271-77. doi: 10.1001/ archinternmed.2009.507.

10. Krebs EE, Gravely A, Nugent S, et al. Effect of opioid vs nonopioid medications on painrelated function in patients with chronic back pain or hip or knee osteoarthritis pain: The SPACE randomized clinical trial. JAMA 2018;319(9):872-82. doi: 10.1001/jama.2018.0899.

11. Traeger A, Buchbinder R, Harris I, Maher C. Diagnosis and management of low-back pain in primary care. CMAJ 2017;189(45):E1386-95. doi: 10.1503/cmaj.170527.

12. Atlas SJ, Deyo RA. Evaluating and managing acute low back pain in the primary care setting. J Gen Intern Med 2001;16(2):120-31.

13. Sears ED, Caverly TJ, Kullgren JT, et al. Clinicians' perceptions of barriers to avoiding inappropriate imaging for low back pain - Knowing is not enough. JAMA Intern Med 2016;176(12):1866-68. doi: 10.1001/jamainternmed.2016.6364.

14. Darlow B, Dean S, Perry M, Mathieson F, Baxter GD, Dowell A. Acute low back pain management in general practice: Uncertainty and conflicting certainties. Fam Pract 2014;31(6):723-32. doi: 10.1093/fampra/cmu051.

15. Karran EL, Medalian Y, Hillier SL, Moseley GL. The impact of choosing words carefully: An online investigation into imaging reporting strategies and best practice care for low back pain. Peer 2017;5:e4151. doi: 10.7717/peerj.4151.

16. Department of Health. Medicare Benefits Schedule book category 5. Canberra: DoH, 2013.

17. Rolfe A, Burton C. Reassurance after diagnostic testing with a low pretest probability of serious disease: Systematic review and meta-analysis. JAMA Intern Med 2013;173(6):407-16. doi: 10.1001/ jamainternmed.2013.2762. 
18. Karran EL, Yau Y, Hillier S, Moseley GL. The reassuring potential of spinal imaging results: Development and testing of a brief, psychoeducation intervention for patients attending secondary care. Eur Spine J 2018;27(1):101-08. doi: 10.1007/s00586-017-5389-8.

19. Brinkikji W, Leutmer $P$, Comstock B, et al. Systematic literature review of imaging features of spinal degeneration in asymptomatic populations. AJNR Am J Neuroradiol 2014;36(4):811-16. doi: 10.3174/ajnr.A4173.

20. Suri P, Boyko EJ, Goldberg J, Forsberg CW, Jarvik JG. Longitudinal associations between incident lumbar spine MRI findings and chronic low back pain or radicular symptoms: Retrospective analysis of data from the longitudinal assessment of imaging and disability of the back (LAIDBACK). BMC Musculoskelet Disord 2014;15:152. doi: 10.1186/1471-2474-15-152.

21. Butler DS, Moseley GL. Explain pain. Portugal: Noigroup Publications, 2003.

22. Vlaeyen JW, Linton SJ. Fear-avoidance model of chronic musculoskeletal pain: 12 years on. Pain 2012;153(6):1144-47. doi: 10.1016/j. pain.2011.12.009.
23. Zusman M. Structure-oriented beliefs and disability due to back pain. Aust J Physiother 1998;44(1):13-20.

24. Roland M, van Tulder M. Should radiologists change the way they report plain radiography of the spine? Lancet 1998;352(9123):229-30.

25. Fried JG, Andrew AS, Ring NY, Pastel DA. Changes in primary care health care utilization after inclusion of epidemiologic data in lumbar spine MR imaging reports for uncomplicated low back pain. Radiology 2018;287(2):563-69. doi: 10.1148/radiol.2017170722

26. Petrie KJ, Müller JT, Schirmbeck F, et al. Effect of providing information about normal test results on patients' reassurance: Randomised controlled trial. BMJ. 2007;334(7589):352

27. O'Connell NE, Cook CE, Wand BM, Ward SP. Clinical guidelines for low back pain: A critical review of consensus and inconsistencies across three major guidelines. Best Pract Res Clin Rheumatol 2016;30(6):968-80. doi: 10.1016/j. berh.2017.05.001. 